\title{
Characteristics and outcomes of patients with postoperative cardiovascular pseudoaneurysms
}

\author{
Michael Chenier, MD, MPH, ${ }^{a}$ Krishna K. Patel, MD, ${ }^{a}$ Lars G. Svensson, MD, PhD, ${ }^{b}$ Jose Navia, MD, ${ }^{b}$

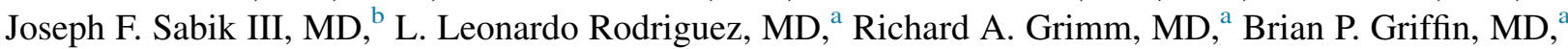 \\ and Milind Y. Desai, MD
}

\begin{abstract}
Background: Data regarding long-term outcomes in cardiovascular pseudoaneurysms are sparse. In patients with prior cardiovascular surgery, we sought to assess characteristics and outcomes in patients with cardiovascular pseudoaneurysms (excluding ischemic left ventricular pseudoaneurysms).

Methods: We included 114 patients (mean age, $57 \pm 16$ years; $70 \%$ were men) with prior cardiovascular surgery who presented with paravalvular $(\mathrm{n}=71,59$ aortic and 12 mitral valve) and ascending aortic $(n=43)$ pseudoaneurysms ( $27 \%$ with systemic infection). Baseline, surgical, and follow-up data were recorded. A composite end point of stroke or death during follow-up was recorded.
\end{abstract}

Results: Additive European System for Cardiac Operative Risk Evaluation was high $(10 \%)$, and $81 \%$ of patients underwent another redo cardiovascular surgery. Over a mean follow-up period of $5.2 \pm 4$ years, there were 37 (32\%) composite events (32 deaths and 5 strokes). Within the surgical subgroup, there were 10 (11\%) composite events during the in-hospital stay. The 1-, 2-, 5-, and 10-year freedom from composite events were $86 \%, 82 \%, 74 \%$, and $55 \%$, respectively. Additive European System for Cardiac Operative Risk Evaluation (hazard ratio, 1.14) and presence of a documented systemic infection (hazard ratio, 3.90) were associated with a higher rate of composite events, whereas subsequent cardiovascular surgery as a time-dependent covariate hazard ratio (hazard ratio, 0.30 ) was associated with improved freedom from composite events (all $P<.05$ ).

Conclusions: Patients with a history of cardiovascular surgery in whom cardiovascular pseudoaneurysms subsequently develop have a high rate of short- and longterm adverse events. A higher additive European System for Cardiac Operative Risk Evaluation and documented systemic infection were associated with a higher rate of composite events, whereas cardiovascular surgery (to correct cardiovascular pseudoaneurysms) during follow-up was associated with improved freedom from adverse events. (J Thorac Cardiovasc Surg 2017;153:43-50)

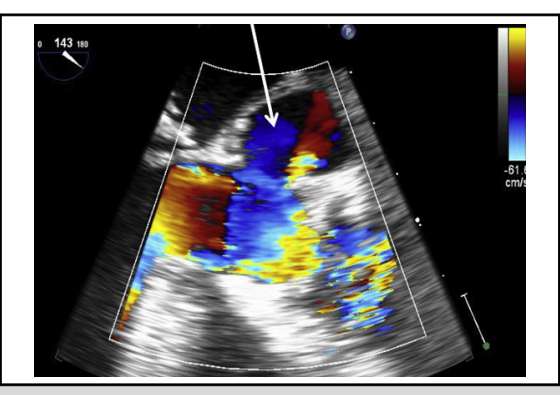

Survival analysis demonstrating worse outcomes in the CV-PSA group versus matched controls.

\section{Central Message}

In a study of 114 patients (mean age, 57 years $70 \%$ were men) with prior $\mathrm{CV}$ surgery who presented with CV-PSA, we demonstrate that there were $37(32 \%)$ events ( 32 deaths and 7 strokes) at $5.2 \pm 4$ years of follow-up. The 1-, 2-, 5 and 10-year freedom from composite events were $86 \%, 82 \%, 74 \%$, and $55 \%$, respectively Higher euroSCORE (HR, 1.14) and systemic infection $(H R, 3.90)$ were associated with higher events, whereas CV surgery $(\mathrm{HR}, 0.30)$ was associated with freedom from events (all $P<.05$ ).

\section{Perspective}

Patients with a history of CV surgery who subsequently develop CV-PSA have a high rate of short- and long-term adverse events. A higher additive euroSCORE and documented systemic infection were associated with a higher rate of composite events, whereas CV surgery (to correct CV-PSA) during follow-up was associated with improved freedom from adverse events.

See Editorial Commentary page 51.

\footnotetext{
From the Heart Valve Center, Departments of ${ }^{a}$ Cardiovascular Medicine and ${ }^{\mathrm{b}}$ Cardiothoracic Surgery, Heart and Vascular Institute, Cleveland Clinic, Cleveland, Ohio.

M.C. and K.P. contributed equally to the work.

Received for publication June 21, 2016; revisions received Aug 18, 2016; accepted for publication Aug 30, 2016; available ahead of print Oct 5, 2016.

Address for reprints: Milind Y. Desai, MD, Department of Cardiovascular Medicine,

Heart and Vascular Institute, Cleveland Clinic, 9500 Euclid Ave, Desk J1-5,

Cleveland, OH 44195 (E-mail: desaim2@ccf.org).

0022-5223/\$36.00

Copyright (c) 2016 by The American Association for Thoracic Surgery

http://dx.doi.org/10.1016/j.jtcvs.2016.08.065
}

Pseudoaneurysms (PSAs) after cardiovascular (CV) surgery are uncommon, often occurring adjacent to a prosthetic valve or at anastomotic sites of aortic grafts. These are

Scanning this QR code will take you the videos for the article.

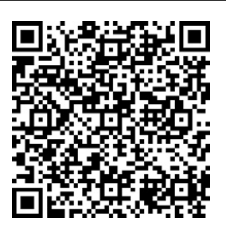




$$
\begin{aligned}
& \text { Abbreviations and Acronyms } \\
& \mathrm{AV} \quad=\text { aortic valve } \\
& \mathrm{CV}=\text { cardiovascular } \\
& \text { euroSCORE }=\text { European System for Cardiac } \\
& \text { Operative Risk Evaluation } \\
& \text { HR = hazard ratio } \\
& \mathrm{LV} \quad=\text { left ventricular } \\
& \text { PSA } \quad=\text { pseudoaneurysm }
\end{aligned}
$$

potentially serious complications that can be fatal and often will lead to a repeat surgery once discovered. CV-PSAs occur when a disruption to 1 or more layers of the wall is contained by the remaining intact layers or adjacent tissues (Figure 1; Videos 1 and 2). This is different from a true aneurysm, which is a vascular dilatation in which all 3 layers (intima, media, and adventitia) are intact without disruption of the vascular wall. The cause of CV-PSA formation after cardiac surgery remains unclear. Postoperative endocarditis or infection most often has been described as the likely culprit, ${ }^{1,2}$ although the presence of postoperative valvular or paravalvular aortic insufficiency, ${ }^{3}$ inadequate bioprosthetic tissue fixation with native tissue degeneration secondary to local immune responses, ${ }^{4,5}$ and poor tissue healing after the trauma of surgery (especially along suture lines or aortic cannulation sites) have been proposed as potential mechanisms.

Data are currently sparse regarding long-term outcomes in patients with CV-PSA that occur after CV surgery because limited instances are reported in small case series or case reports in the literature. ${ }^{6-11}$ In patients with prior CV surgery, we sought to assess the clinical characteristics and long-term outcomes in patients with documented CV-PSAs (defined as paravalvular, aortic root, or ascending aorta PSAs).

\section{MATERIALS AND METHODS Study Sample}

This was an observational cross-sectional study of 114 patients (aged $\geq 18$ years), with a history of CV surgery and a confirmed diagnosis of CV-PSA, who were evaluated at the Cleveland Clinic between 1996 and 2013 for further management of their clinical condition, including consideration of possible redo cardiac surgery. Prior CV surgery was defined as having undergone aortic root/ascending aortic repair/ replacement, aortic valve (AV) repair/replacement, mitral valve repair/ replacement, tricuspid valve repair/replacement, or a combination of the valvular repair or replacements noted (with or without concomitant coronary artery bypass grafting). Clinical and demographic data were prospectively entered into the electronic medical records at the time of initial visit. The need for repeat surgical procedures was ascertained. The study was approved by our institutional review board. On the basis of available data, an additive European System for Cardiac Operative Risk Evaluation (euroSCORE) was calculated in each patient. ${ }^{12}$ Although such scores have been developed to predict perioperative mortality, we
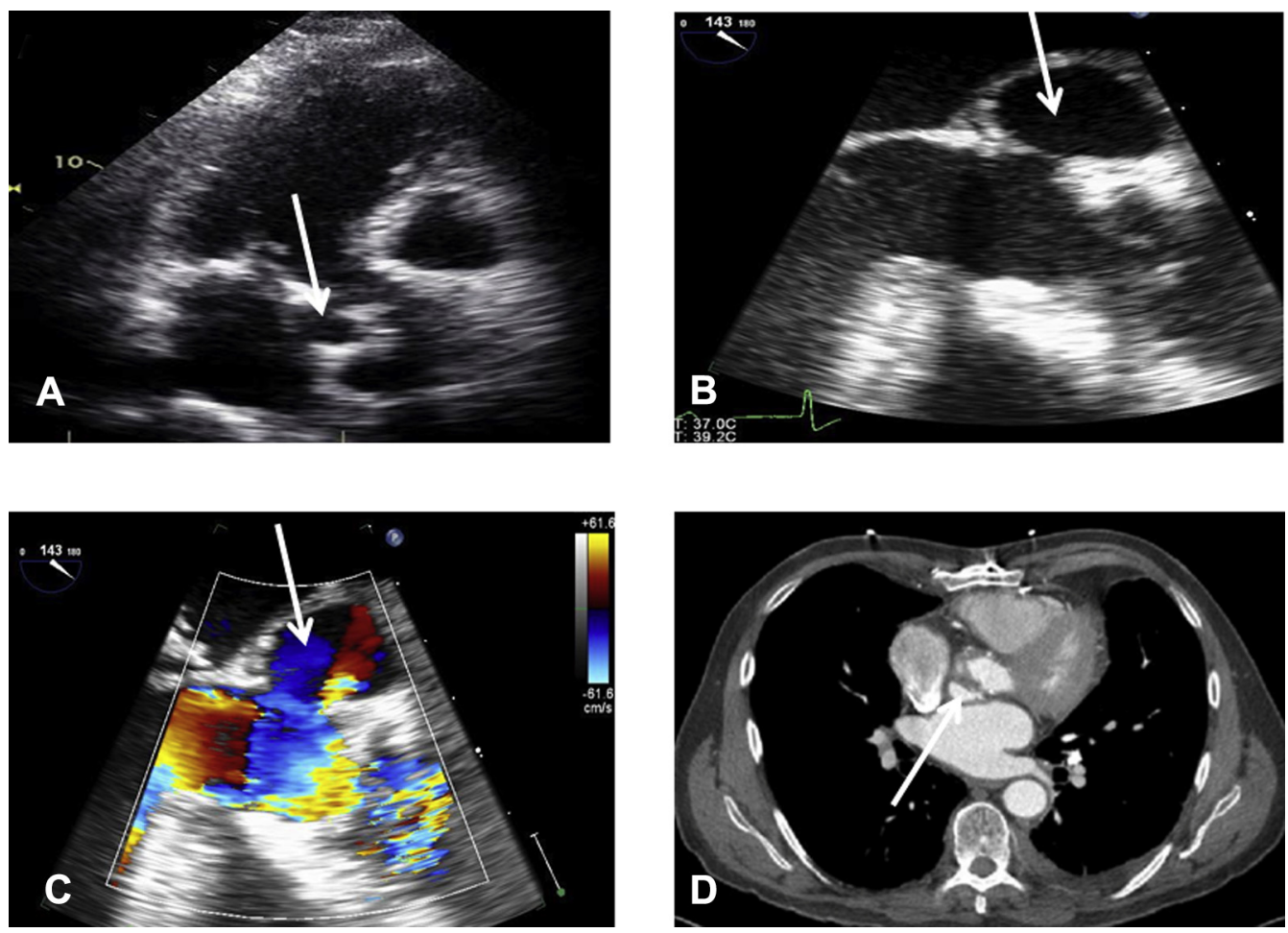

FIGURE 1. An example of a patient with a history of bioprosthetic AV replacement and an infected aortic root PSA with a fistulous connection (arrow) on 3-chamber transthoracic echocardiogram (A), 2-dimensional and color Doppler transesophageal echocardiogram (B and C), and contrast-enhanced computed tomography (D). 


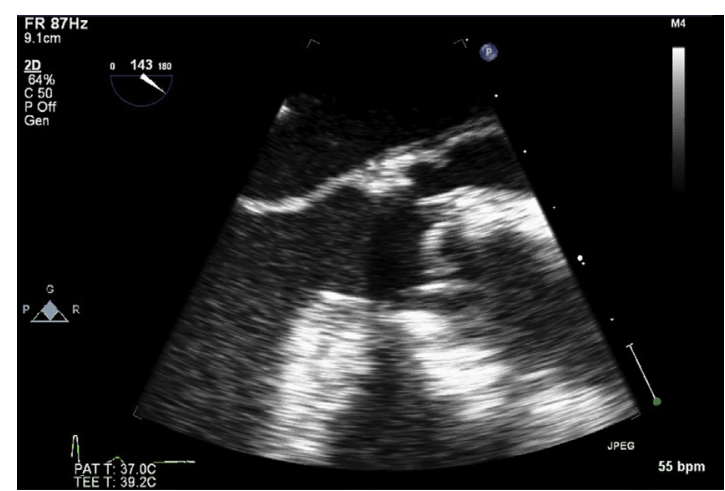

VIDEO 1. Two-dimensional transesophageal echocardiographic image of the same patient as in Figure 1 with a history of bioprosthetic AV replacement and an infected aortic root PSA with a fistulous connection. Video available at: http://www.jtcvsonline.org/article/S0022-5223(16)311 35-7/addons.

chose to calculate the euroSCORE because it represents a composite of various predictors that are known to be associated with outcomes in such patients. The study investigators performed a manual review of the electronic charts and imaging database to ensure that all clinical and imaging data were present before analysis.

CV-PSA was identified from our imaging (echocardiographic and tomographic) database and defined as valvular (aortic or mitral) or involving the aortic root or ascending aorta. We excluded PSAs of the left ventricular (LV) cavity, associated with ischemic heart disease, because these have a different pathophysiologic profile. Patients with a "confirmed CV-PSA" were identified after a thorough analysis of the electronic medical records and multimodality imaging data (Figure 1; Videos 1 and 2). To conclusively ascertain the diagnosis of CV-PSA, 79 patients had a transesophageal echocardiogram, 78 patients had a cardiac computed tomography, and 23 patients had a cardiac magnetic resonance performed in conjunction with the initial transthoracic echocardiogram.

\section{Imaging Data Acquisition}

Transthoracic echocardiography. All patients underwent a comprehensive echocardiogram with commercially available instruments (Philips Medical Systems, NA, Bothell, Wash; General Electric Medical Systems, Milwaukee, Wis; and Siemens Medical Solutions USA, Inc, Malvern, Pa) as part of a standard clinical diagnostic evaluation.

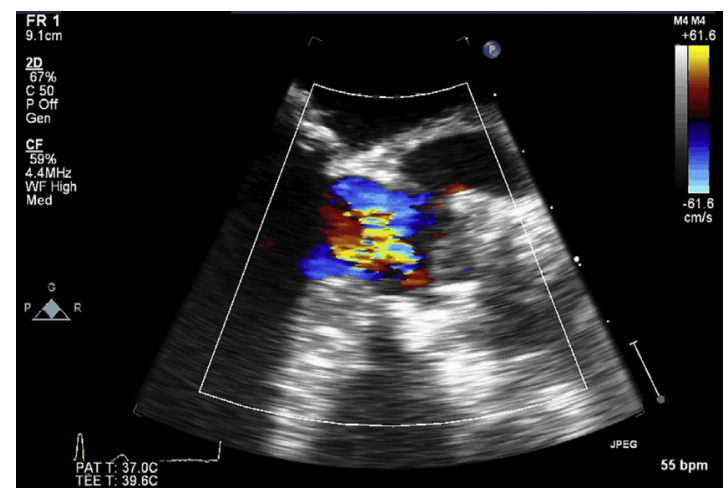

VIDEO 2. Color Doppler transesophageal echocardiographic image of the same patient as in Figure 1 with a pulsatile aortic root PSA and color flow suggestive of a fistulous connection with LV outflow tract. Video available at: http://www.jtcvsonline.org/article/S0022-5223(16)31135-7/addons.
Measurements and recordings were obtained according to the American Society of Echocardiography recommendations. ${ }^{13,14} \mathrm{LV}$ and left atrial dimensions were measured from the 2-dimensional echocardiogram. LV ejection fraction was calculated using the Simpson's biplane method. For quantification of aortic stenosis, the LV outflow tract diameter was measured on parasternal long-axis views. Pulsed-wave and continuous-wave Doppler were used to record velocities across the LV outflow tract and AV, respectively. The AV was examined from multiple windows including apical, suprasternal notch, and right parasternal to obtain the peak $\mathrm{AV}$ velocity and mean $\mathrm{AV}$ gradient. A semiquantitative 5-point scale (none to severe) was used to stratify valvular regurgitation, assessed by color 2-dimensional Doppler echocardiography clips obtained in apical 2- and 4-chamber views, according to guidelines. Right ventricular systolic pressure was estimated in a standard fashion. ${ }^{15}$

Advanced imaging. As discussed, all patients with CV-PSA underwent at least 1 additional advanced imaging technique to confirm the diagnosis of CV-PSA. Multiplane transesophageal echocardiography (commercially available equipment similar to machines used for transthoracic echocardiography) was performed under conscious sedation in a standard fashion. Contrast-enhanced computed tomography (Philips Medical Systems, Best, The Netherlands, or Siemens Medical Solutions, Erlangen, Germany) of the chest was performed using electrocardiographic gated (spiral retrospective or prospectively triggered axial acquisition) protocols in a standard fashion using 2-mm slice thickness. Likewise, a cardiac magnetic resonance study was performed (Philips Medical Systems or Siemens Medical Solutions) using typical sequences including balanced steady-state free precession (cine) images in multiple (short-axis, 2-, 3-, and 4-chamber) views.

\section{Definition and Follow-up of Cardiovascular \\ Pseudoaneurysms}

CV-PSA was defined as a fluid-filled cavity adjacent to various CV structures with a free communication with the CV circuit. CV-PSA adjacent to the prosthetic valve was most commonly due to an infectious process and represented an abscess cavity (Figure 1; Videos 1). CV-PSA adjacent to the aortic root/ascending aorta was a fluid-filled cavity most commonly seen at the anastomosis of surgical grafts and could be due to an infectious process (abscess) or simple breakdown at the anastomotic site (blood-filled cavity). These structures appeared pulsatile with a free communication with the CV circuit on Doppler echocardiography or contrast-filled cavity seen on tomographic images. The CV-PSAs were broadly divided into 2 categories: infectious and noninfectious (deemed to be due to a technical issue, such as suture dehiscence at the aortic graft anastomotic site). All patients with CV-PSA with evidence of systemic infection at the initial evaluation or reemergence of infection during follow-up were deemed unstable and were recommended surgery to prevent rupture and provide infection source control. However, in the documented noninfectious patients, surgery was only recommended for unstable patients, defined as (1) CV-PSA $1 \mathrm{~cm}$ or more in size at initial presentation or (2) rapid growth on serial imaging $(>0.5 \mathrm{~cm}$ in a 6-month period). Patients with noninfectious CV-PSAs that remained stable on serial imaging were not offered surgery. In addition, patients with CV-PSAs (including infectious) who were deemed to be high risk (because of advanced comorbidities) for redo cardiac surgery were not offered surgery.

\section{Surgical Details}

The following surgical procedures were undertaken: (1) aortic or mitral valve repair/replacement; (2) aortic or mitral valve repair/ replacement + coronary bypass grafting; (3) ascending aortic replacement alone; and (4) aortic/mitral valve repair/replacement + ascending aortic replacement \pm coronary bypass grafting. We did not use BioGlue (CryoLife, Kennesaw, Ga) during the repair of CV-PSA. In case of a known infection, we typically performed an extensive surgery for infection source control. In composite AV + aortic replacement (Bentall procedure), we approached the coronary reimplantation in 2 different approaches: 
(1) coronary button reimplantation technique, in which the coronary ostia were completely excised and mobilized as buttons of the aorta, followed by sewing them to the openings in the Dacron graft along with bovine pericardial strip or Teflon felt to reinforce and cushion the anastomosis suture line to avoid bleeding; or (2) the Cabrol technique, in which an 8-mm Dacron interposition tube graft was sewn end-to-end to the right and left coronary ostia, and then with a side-to-side anastomosis to the aortic graft. Also, we did not perform off-pump partial repair of the PSA to perform a complete surgical elimination of the false aneurysm and reconstruct the anatomic area to avoid future surgical intervention and avoid the potential of developing infections.

\section{Outcomes Assessment}

The follow-up period started from the time of baseline echocardiography that detected the CV-PSA at the Cleveland Clinic to the last follow-up or death. We recorded all-cause mortality from the medical records, Social Security Death Index, and Ohio death index, and reconfirmed this by inspection of the death certificate or verification with a family member. We also recorded stroke during follow-up. Stroke was defined as neurologic impairment lasting more than 24 hours because of cerebral ischemia or hemorrhage confirmed by radiographic studies. Transient ischemic attack was similarly defined as neurologic impairment lasting less than 24 hours. A composite primary outcome of stroke or death was recorded. In addition, for the patients who underwent redo $\mathrm{CV}$ surgery during the follow-up period, we also collected additional clinical details of the perioperative period, including length of hospital stay, major perioperative bleeding, ${ }^{16}$ and need for redo surgery (mediastinal bleeding being the most common cause) during the same hospital stay.

\section{Statistical Analysis}

Patient and procedural characteristics were expressed as mean (standard deviation) if approximately parametrically distributed or median (interquartile range) if nonparametrically distributed. Categoric data were expressed as a percentage. To assess outcomes, multivariable Cox proportional hazards analysis was used. Relevant clinical and echocardiographic variables that are known to be associated with outcomes in such patients were considered for the survival analysis, avoiding collinearity and overfitting. CV surgery was included as a timedependent covariate in Cox survival analysis. For each patient undergoing CV surgery, the analysis time was modeled so that only the person-time after surgery was included in follow-up for survival analysis. Time before surgery (but after the baseline echocardiogram) was not included in follow-up for survival analysis. In the subgroup survival analysis of only those patients who underwent CV surgery during follow-up, the date of surgery was considered as time zero for the beginning of follow-up. Hazard ratios (HRs) with $95 \%$ confidence intervals were calculated. Survival curves for cumulative proportion of events as a function over time were obtained. Statistical analysis was performed using SPSS version 11.5 (SPSS Inc, Chicago, Ill) and Stata version 10.0 (StataCorp LP, College Station, Tex).

\section{RESULTS}

Baseline clinical and demographic data of the study sample are shown in Table 1. As would be expected from a study sample of patients being considered for redo CV surgery, the mean additive euroSCORE was $10 \pm 3$, suggesting a higher than usual risk. In addition, 31 patients $(27 \%)$ in the CV-PSA group had evidence of systemic infection, with the breakdown of organisms as follows: 8 methicillin-sensitive Staphylococcus aureus, 1 methicillin-
TABLE 1. Baseline characteristics of the study sample $(n=114)$

\begin{tabular}{lc}
\hline \multicolumn{1}{c}{ Variable } & \\
\hline Age (y) & $57 \pm 16$ \\
Female gender & $34(30)$ \\
Hypertension & $61(54)$ \\
Diabetes mellitus & $15(13)$ \\
Hyperlipidemia & $36(32)$ \\
Coronary artery disease & $10(9)$ \\
Atrial fibrillation & $29(25)$ \\
Stroke & $15(13)$ \\
Severe chronic kidney disease & $10(9)$ \\
Additive euroSCORE & $10 \pm 2$ \\
Beta-blockers & $81(71)$ \\
ACEIs & $40(35)$ \\
Statins & $40(35)$ \\
No. of previous CV surgeries & \\
$\quad 1$ & $57(50)$ \\
$\geq 2$ & $57(50)$ \\
Time from previous CV surgery to current & $5 \pm 6$ \\
$\quad$ clinical evaluation (y) & \\
Type of prior CV surgery & \\
AV/MV repair/replacement \pm ascending & $110(96)$ \\
$\quad$ aortic replacement & \\
AV/MV repair/replacement + CABG & $4(4)$ \\
Evidence of systemic infection (positive blood cultures) & $31(27 \%)$ \\
\hline Con &
\end{tabular}

Continuous variables reported as mean \pm standard deviation, and categoric variables expressed as n (\%). euroSCORE, European System for Cardiac Operative Risk Evaluation; $A C E I$, angiotensin receptor blocker inhibitor; $C V$, cardiovascular; $A V$, aortic valve; $M V$, mitral valve; $C A B G$, coronary artery bypass grafting.

resistant $S$ Aureus, 3 coagulase-negative Staphylococcus, 2 methicillin-sensitive $S$ epidermis, 2 Enterococcus fecalis, 2 Streptococcus viridans, 1 Escherichia coli, 2 Propionibacterium acnes, 3 Candida albicans, 1 Acinetobacter, and 5 no single organism identified.

Baseline echocardiographic characteristics of the study sample are shown in Table 2. A total of 59 patients had a CV-PSA associated with the AV, 12 with the mitral valve and 43 with the aortic root or ascending aorta. All of these patients had at least 1 additional imaging study to confirm the presence of CV-PSA.

\section{Surgical Follow-up}

After the diagnosis of CV-PSA, 92 patients $(81 \%)$ underwent redo $\mathrm{CV}$ surgery at $26 \pm 30$ days during follow-up, when the presence of CV-PSA was confirmed. The proportion of various CV surgeries performed were as follows: (1) aortic or mitral valve repair/replacement (6 [5\%]); (2) aortic or mitral valve repair/replacement + coronary bypass grafting (1[1\%]); (3) ascending aortic replacement alone (18 [16\%]); and (4) aortic/mitral valve repair/replacement + ascending aortic replacement \pm coronary bypass grafting (67 [59\%]). Within group 4, 17 patients had a composite AV + ascending aortic graft (Bentall procedure) with coronary reimplantation. Of these 17 patients, coronary button reimplantation technique was 
TABLE 2. Echocardiographic data of the study sample $(n=114)$

\begin{tabular}{lc}
\multicolumn{1}{c}{ Variable } \\
\hline LV ejection fraction $(\%)$ & $53 \pm 9$ \\
Left atrial diameter $(\mathrm{cm})$ & $4.3 \pm 0.7$ \\
Mean gradient across AV $\geq 40 \mathrm{~mm} \mathrm{Hg}$ & 0 \\
Aortic regurgitation & \\
$\quad$ None & $67(59)$ \\
Mild & $22(19)$ \\
Moderate & $11(10)$ \\
Moderate-severe & $7(6)$ \\
Severe & $7(6)$ \\
Mitral regurgitation & \\
None & $57(50)$ \\
Mild & $31(27)$ \\
Moderate & $15(13)$ \\
Moderate-severe & $7(6)$ \\
Severe & $4(4)$ \\
Tricuspid regurgitation & \\
None & $69(61)$ \\
Mild & $24(21)$ \\
Moderate & $11(10)$ \\
Moderate-severe & $6(5)$ \\
Severe & $4(4)$ \\
Right ventricular systolic pressure (mm Hg) & $31 \pm 18$ \\
\hline Continuous variables reported as mean \pm standard deviation, and categoric variables \\
expressed as \%. $L V$, Left ventricular; $A V$, aortic valve.
\end{tabular}

used in 13 patients, and the Cabrol technique was used in 4 patients. The details of why 22 patients were not offered surgery are listed in Table 3. Of these patients, $12(55 \%)$ were turned down for CV surgery because of prohibitive perioperative risk or major comorbidities, and they died during follow-up. The other 10 patients $(45 \%)$ within this subgroup were not offered surgery because the CV-PSA had remained stable during serial imaging follow-up. None of these patients have died during follow-up, and the CV-PSA has remained stable on multimodality imaging.

In the study sample, 17 patients (18\%) had documented major bleeding in the perioperative period. Likewise, 10 patients $(11 \%)$ in the CV-PSA group required a redo operation during the same surgical hospitalization for significant internal bleeding. The in-hospital length of stay for the current study population was high at $20 \pm 15$ days. At the time of discharge, 34 patients $(30 \%)$ were discharged with appropriate intravenous antibiotics, based on culture sensitivity (lasting for at least a 1-week posthospital discharge). No patient has required additional surgery during follow-up. Also, there was no evidence of inflammatory aortitis, foreign body birefringent reactions, or tuberculosis on histopathologic staining.

\section{Outcomes}

Over a mean follow-up period of $5.2 \pm 4$ years, there were $37(32 \%)$ composite events (32 deaths and 5 strokes). Within the subgroup of patients who underwent CV surgery $(\mathrm{n}=92)$, there were $10(11 \%)$ composite events at 30 days
TABLE 3. Reasons for patients with cardiovascular pseudoaneurysms not undergoing redo cardiac surgery during follow-up and their outcomes

\begin{tabular}{|c|c|c|}
\hline Patient & Reason for no surgery during follow-up & $\begin{array}{l}\text { Dead or } \\
\text { alive }\end{array}$ \\
\hline 1 & $\begin{array}{l}\text { Turned down because of comorbidities, } \\
\text { died of ruptured aorta }\end{array}$ & Dead \\
\hline 2 & $\begin{array}{l}\text { Turned down because of comorbidities, } \\
\text { died of dissection and ruptured aorta }\end{array}$ & Dead \\
\hline 3 & $\begin{array}{l}\text { History of Marfan's, turned down because of } \\
\text { paraplegia, died of ruptured aorta }\end{array}$ & Dead \\
\hline 4 & Stable CV-PSA, disease not advanced enough & Alive \\
\hline 5 & Stable CV-PSA, disease not advanced enough & Alive \\
\hline 6 & Turned down because of lung cancer & Dead \\
\hline 7 & Turned down because of malignant melanoma & Dead \\
\hline 8 & Stable CV-PSA, disease not advanced enough & Alive \\
\hline 9 & Stable CV-PSA, disease not advanced enough & Alive \\
\hline 10 & $\begin{array}{l}\text { Turned down because of comorbidities and } \\
\text { morbid obesity, died of sepsis }\end{array}$ & Dead \\
\hline 11 & $\begin{array}{l}\text { Turned down because of comorbidities, died } \\
\text { of sepsis }\end{array}$ & Dead \\
\hline 12 & Stable CV-PSA, disease not advanced enough & Alive \\
\hline 13 & Stable CV-PSA, disease not advanced enough & Alive \\
\hline 14 & $\begin{array}{l}\text { Turned down because of comorbidities, died } \\
\text { of sepsis }\end{array}$ & Dead \\
\hline 15 & Stable CV-PSA, disease not advanced enough & Alive \\
\hline 16 & Stable CV-PSA, disease not advanced enough & Alive \\
\hline 17 & $\begin{array}{l}\text { Turned down because of comorbidities, died } \\
\text { of sepsis }\end{array}$ & Dead \\
\hline 18 & $\begin{array}{l}\text { Turned down because of comorbidities, died } \\
\text { of sepsis }\end{array}$ & Dead \\
\hline 19 & Stable CV-PSA, disease not advanced enough & Alive \\
\hline 20 & Stable CV-PSA, disease not advanced enough & Alive \\
\hline 21 & $\begin{array}{l}\text { Turned down because of comorbidities, died } \\
\text { of sepsis }\end{array}$ & Dead \\
\hline 22 & $\begin{array}{l}\text { Turned down because of comorbidities, died } \\
\text { of sepsis }\end{array}$ & Dead \\
\hline
\end{tabular}

CV-PSA, Cardiovascular pseudoaneurysms.

during the in-hospital stay. Also, 1-, 2-, 5-, and 10-year freedom from composite events were $86 \%, 82 \%, 74 \%$ and $55 \%$, respectively. The survival curve of the study sample is shown in Figure 2.

The results of multivariable Cox proportional survival analysis are shown in Table 4. The additive euroSCORE (HR, 1.14) and presence of a documented systemic infection (HR, 3.90) were associated with a higher rate of composite events, whereas subsequent CV surgery, as a time-dependent covariate HR (HR, 0.30), was associated with improved freedom from composite events (all $\mathrm{P}<.05$ ).

Because a substantial proportion of patients in the CV-PSA group were turned down for CV surgery because of advanced disease or comorbidities (Table 3), we further performed survival analysis after excluding patients who did not undergo CV surgery. Within this group of 92 patients, there were $24(26 \%)$ composite events. 


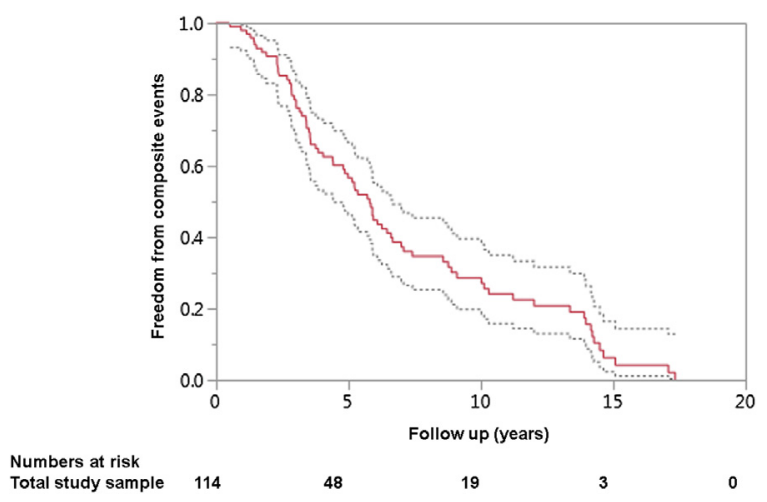

FIGURE 2. Survival analysis of the study sample.

Multivariable Cox proportional hazards survival analysis demonstrated that additive euroSCORE (HR, 1.10; $1.02-1.18 ; P=.03)$ and presence of a documented systemic infection (HR, $4.23 ; 1.21-11.58 ; P=.02)$ were associated with a higher rate of composite events. The survival curve of the subgroup that underwent $\mathrm{CV}$ surgery during follow-up is shown in Figure 3.

Per our institutional protocol, all patients who underwent an operation for CV-PSA underwent follow-up echocardiography before discharge. Subsequently, they were followed periodically using a combination of echocardiography and tomographic imaging at the discretion of the treating cardiologist. None of the operated patients had a recurrence of CV-PSA or infection.

\section{DISCUSSION}

In the current study, we describe the characteristics and long-term outcomes of patients with a history of CV surgery and a documented CV-PSA (paravalvular and aortic, excluding those occurring in the LV due to ischemic heart disease). As expected, because of a history of CV surgery, all patients in the study sample were considered high risk, as evidenced by a high additive euroSCORE. The high-risk status was further confirmed by the observation that approximately 1 in 3 patients had a composite event during follow-up, with 1-, 5-, and 10-year survival of

TABLE 4. Multivariable Cox proportional hazards survival analysis in the study sample

\begin{tabular}{lcc}
\hline \multicolumn{1}{c}{ Variable } & HR & $\boldsymbol{P}$ value \\
\hline Additive euroSCORE & $1.14(1.03-1.26)$ & .01 \\
Documented systemic infection & $3.90(1.21-7.92)$ & .02 \\
CV surgery during follow-up & $0.30(0.15-0.63)$ & .001 \\
$\quad$ (time-dependent covariate) & & \\
\hline
\end{tabular}

Chi-square for the model was $19, P<.001$. The following variables were considered for the survival analysis: Additive euroSCORE, documented systemic infection, degree of aortic and mitral regurgitation, and CV surgery during follow-up. Variables that were included in the calculation of additive euroSCORE were not individually considered for survival analysis. HR, Hazard ratio; euroSCORE, European System for Cardiac Operative Risk Evaluation; $C V$, Cardiovascular.

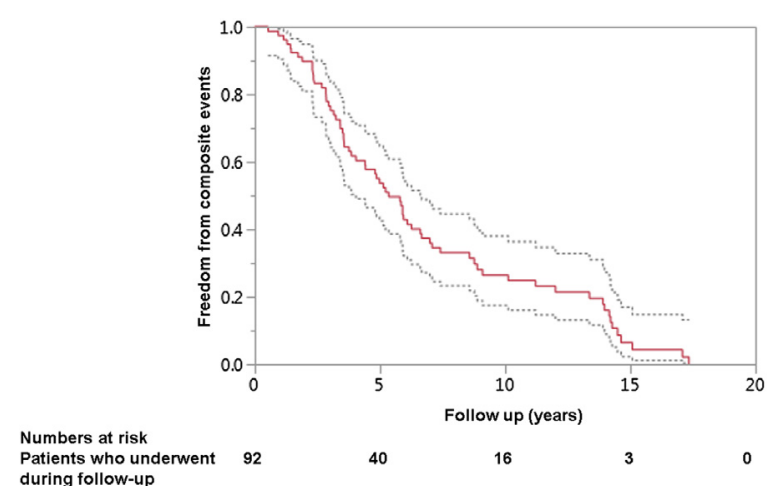

FIGURE 3. Survival analysis of subgroup of the study sample that underwent surgery during follow-up.

$86 \%, 74 \%$, and $55 \%$, respectively. A higher euroSCORE and evidence of systemic infection were independently associated with a significantly higher rate of composite events, whereas surgical correction of CV-PSA was associated with an improved long-term freedom from composite events.

Approximately $20 \%$ of patients did not undergo CV surgery during follow-up, with $55 \%$ of those being turned down because of high perioperative risk or major comorbidities (Table 3). The other $45 \%$ did not undergo CV surgery because the CV-PSA was deemed stable on serial monitoring. As expected, all patients who were turned down for surgery died during follow-up. When only the subgroup of CV-PSA undergoing CV surgery during follow-up was studied, higher additive euroSCORE and systemic infection were independently associated with adverse events.

CV-PSA formation after CV surgery is rare, with recent studies demonstrating an incidence of less than $1 \%$ for aortic PSAs. ${ }^{7,17}$ The incidence of CV-PSA formation specifically after valvular surgery remains unclear. As early as the 1950s, postoperative PSAs of the ascending aorta due to surgical trauma associated with aortic incisions and suture lines were identified as potentially fatal complications of CV surgery. ${ }^{18-20}$ Although tuberculosis has long been recognized as a cause for aortic PSA formation, ${ }^{21-23}$ studies have identified postoperative nontuberculin infection as a potential contributor to PSA formation. ${ }^{1,24}$ Sullivan and colleagues ${ }^{1}$ identified a majority of patients with postoperative PSAs (18/31 in their review) having "confirmed or highly suspected active infection" after CV surgery. Similar studies in the literature observed the same general findings, and during that era some surgical centers advocated for aortography to evaluate for the presence of a PSA in patients with sustained bacteremia after cardiac surgery before tomographic imaging had become a mainstay. ${ }^{25}$ At the Cleveland Clinic, CV-PSAs associated with a systemic infection are identified on $\mathrm{CV}$ multimodality imaging (surface or transesophageal 
echocardiography, computed tomography, or magnetic resonance imaging). However, the truly noninfectious CV-PSAs typically are detected incidentally on surveillance imaging. We routinely perform baseline postoperative and serial follow-up imaging (echocardiographic and tomographic) in patients who have undergone complex $\mathrm{CV}$ (including ascending aortic) surgery. If no CV-PSA is identified, patients have periodic surveillance imaging (typically every 1-2 years), but if a CV-PSA is identified, then they would have more frequent surveillance imaging to establish stability or progression.

Since these early descriptions of postoperative CV-PSAs, few data remain regarding clinical outcomes and definitive operative management. One of the largest recent studies observed CV-PSA formation, specifically of the mitral-aortic intervalvular fibrosa, after AV replacement or confirmed infective endocarditis in a limited, systematic review of 149 patients in the literature. ${ }^{6}$ Most of the patients in this review underwent surgical repair of the CV-PSA after diagnosis; however, the clinical outcomes were not well delineated between the studies included in the systematic review and their respective patient groups. Much of the risk of having a CV-PSA historically has been found to be due to CV-PSA rupture with sudden hemodynamic collapse (spontaneous or during attempted surgical repair itself with repeat sternotomy), ${ }^{7,8}$ coronary obstruction, or worsening heart failure with or without significant aortic insufficiency. Because of the often insidious nature that CV-PSAs present, no convincing data on risk factors for CV-PSA formation exist. Pham and colleagues ${ }^{9}$ described the preoperative risk factors for aortic PSA formation after aortic crossclamping as moderate to heavy calcification of the aorta, a clinical history of connective tissue disorders, "any" infection, and surgical "blowout" at the aortotomy site. Enseleit and colleagues ${ }^{17}$ reported that having a composite graft placed was associated with a 27 -fold increase in the risk for postoperative PSA formation compared with AVR alone (regardless of type of prosthetic valve used). Other studies have described a clinical history of endocarditis, mediastinitis, or even asymptomatic bacteremia in the perioperative period as leading to a higher risk of postoperative PSA formation. ${ }^{10,11}$ Atik and colleagues $^{26}$ described 60 cases of aortic PSA repair (almost all, 83\%, had undergone prior cardiac surgery) with a hospital mortality of $6.7 \%$ and long-term survival of $74 \%$ at 5 years and $60 \%$ at 10 years. All of the patients in the study by Atik and colleagues ${ }^{26}$ underwent surgical repair or resection of aortic PSA, a strategy that was recommended by the authors in nearly all cases of aortic PSA formation after prior surgery. However, some publications have demonstrated long-term survival in patients with CV-PSAs who are managed medically without reoperation. ${ }^{27}$ As with the other limitations currently in the literature regarding this diagnosis, the data regarding conservative, nonoperative management of postoperative PSAs consist of small case reports and series.

\section{Clinical Implications}

To the best of our knowledge, our study is one of the largest to systematically report long-term outcomes in patients with a documented CV-PSA. The current study demonstrates that the presence of a CV-PSA, especially if associated with systemic infection, has poor long-term outcomes and that surgery to correct CV-PSA is associated with improved survival. In terms of broader management of documented CV-PSA at the Cleveland Clinic, we divide them into 2 categories: infectious and noninfectious (likely technical issues, such as suture dehiscence at the aortic graft anastomotic site). Typically, all patients with CV-PSA with an evidence of systemic infection at initial evaluation or reemergence of infection during follow-up are recommended to undergo surgery to prevent rupture and infection source control (unless there are prohibitive comorbidities). At the time of surgery, in case of an infected CV-PSA, we typically perform an extensive surgery for infection source control (rather than off-pump procedures). We do not use BioGlue during such surgeries or insert larger prostheses (unless there is concern for patient-prosthesis mismatch, in which situation we enlarge the aortic root). We also perform careful coronary reimplantation in the setting of composite valve + aortic grafting, as discussed earlier. In recent years, with the advent of percutaneous approaches, these could be attempted in carefully selected noninfectious cases. On the other hand, in the documented noninfectious patients, surgery is recommended only for unstable patients, as described earlier. Noninfectious CV-PSAs that remain stable on serial imaging typically are treated conservatively.

\section{Study Limitations}

The retrospective cross-sectional observational nature of the current study had many limitations, as would be expected from such a study design. The current observational study only reports associations, not causality. The current study was not designed to prospectively test the natural history of CV-PSA. For the duration of the study, the sample size is relatively small, and it is seemingly conceivable that our strategy of using transthoracic echocardiography might have missed the CV-PSA, which was subsequently identified intraoperatively. CV-PSA has an infectious cause in a high proportion of patients, and the characteristics and outcomes in these patients are more a reflection of the broader endocarditis milieu. However, not all patients with CV-PSA have an infection-related CV-PSA. Thus, it is crucial to ascertain the cause of the CV-PSA. Also, we report all-cause mortality as the primary end point, as opposed to cardiac 
mortality. However, it has been demonstrated that all-cause mortality is more objective than cardiac mortality. ${ }^{28}$

\section{CONCLUSIONS}

Patients with a history of CV surgery in whom CV-PSA subsequently develops have a high incidence of systemic infection and a high rate of short- and long-term adverse events (death or stroke). In this group, a higher additive euroSCORE and documented systemic infection were associated with a higher rate of composite events, whereas $\mathrm{CV}$ surgery during follow-up was associated with improved freedom from adverse events. These findings need additional confirmation.

\section{Conflict of Interest Statement}

J.F.S. is a consultant for Medtronic and Sorin. All other authors have nothing to disclose with regard to commercial support.

\section{References}

1. Sullivan KL, Steiner RM, Smullens SN, Griska L, Meister SG. Pseudoaneurysm of the ascending aorta following cardiac surgery. Chest. 1988;93:138-43.

2. Sudhakar S, Sewani A, Agrawal M, Uretsky BF. Pseudoaneurysm of the mitral-aortic intervalvular fibrosa (MAIVF): a comprehensive review. J Am Soc Echocardiogr. 2010;23:1009-18; quiz 1112.

3. Smith CR, Stamou SC, Hooker RL, Willekes CC, Heiser JC, Patzelt LH, et al. Stentless root bioprosthesis for repair of acute type A aortic dissection. J Thorac Cardiovasc Surg. 2013;145:1540-54.

4. Englum BR, Pavlisko EN, Mack MC, Ganapathi AM, Schechter MA, Hanna JM, et al. Pseudoaneurysm formation after Medtronic freestyle porcine aortic bioprosthesis implantation: a word of caution. Ann Thorac Surg. 2014;98: 2061-7.

5. David TE, Armstrong S, Maganti M, Butany J, Feindel CM, Bos J. Postimplantation morphologic changes of glutaraldehyde-fixed porcine aortic roots and risk of aneurysm and rupture. J Thorac Cardiovasc Surg. 2009;137: 94-100.

6. Xie M, Li Y, Cheng TO, Wang X, Lu Q, He L, et al. Pseudoaneurysm of the mitral-aortic intervalvular fibrosa. Int J Cardiol. 2013;166:2-7.

7. Emaminia A, Amirghofran AA, Shafa M, Moaref A, Javan R. Ascending aortic pseudoaneurysm after aortic valve replacement: watch the tip of the cardioplegia cannula! J Thorac Cardiovasc Surg. 2009;137:1285-6.

8. Katsumata T, Moorjani N, Vaccari G, Westaby S. Mediastinal false aneurysm after thoracic aortic surgery. Ann Thorac Surg. 2000;70:547-52.

9. Pham N, Zaitoun H, Mohammed TL, DeLaPena-Almaguer E, Martinez F, Novaro GM, et al. Complications of aortic valve surgery: manifestations at CT and MR imaging. Radiographics. 2012;32:1873-92.

10. Grebenc ML, Zech ER. Case 42: aortic homograft anastomotic dehiscence and pseudoaneurysm formation. Radiology. 2002;222:139-43.
11. Parihar B, Choudhary LS, Madhu AP, Alpha MK, Thankachen R, Shukla V. Pseudoaneurysm of ascending aorta after aortic valve replacement. Ann Thorac Surg. 2005;79:705-7.

12. Roques F, Michel P, Goldstone AR, Nashef SA. The logistic EuroSCORE. Eur Heart J. 2003;24:881-2.

13. Lang RM, Bierig M, Devereux RB, Flachskampf FA, Foster E, Pellikka PA, et al. Recommendations for chamber quantification: a report from the American Society of Echocardiography's Guidelines and Standards Committee and the Chamber Quantification Writing Group, developed in conjunction with the European Association of Echocardiography, a branch of the European Society of Cardiology. J Am Soc Echocardiogr. 2005;18:1440-63.

14. Baumgartner H, Hung J, Bermejo J, Chambers JB, Evangelista A, Griffin BP, et al. Echocardiographic assessment of valve stenosis: EAE/ASE recommendations for clinical practice. J Am Soc Echocardiogr. 2009;22:1-23; quiz 101-2.

15. Schwammenthal E, Vered Z, Agranat O, Kaplinsky E, Rabinowitz B, Feinberg MS. Impact of atrioventricular compliance on pulmonary artery pressure in mitral stenosis: an exercise echocardiographic study. Circulation. 2000;102:2378-84.

16. An international randomized trial comparing four thrombolytic strategies for acute myocardial infarction. The GUSTO investigators. N Engl J Med. 1993; 329:673-82.

17. Enseleit F, Grunenfelder J, Braun J, Matthews F, Jenni R, van der Loo B. Formation of pseudoaneurysm after aortic valve replacement without previous endocarditis: a case-control study. J Am Soc Echocardiogr. 2010;23:741-6.

18. Lord JW Jr, Stone PW. False aneurysm of aorta following insertion of a plastic valve for aortic insufficiency. Surgery. 1955;38:593-9.

19. Campbell GS. Injuries of the thoracic aorta. Selective review and case report of repair of false aortic aneurysm thirteen months after aortic valve surgery. Am J Surg. 1963; 105:462-8.

20. Baba N, McKissick TL. Mycotic false aneurysm of the aorta following aortic valvular prosthesis; a case report. Circulation. 1965;31:575-8.

21. Herndon JH, Galt J, Austin DJ. Ruptured tuberculous false aneurysm of the abdominal aorta; report of a case with resection of the aneurysm and survival for six days. Tex State J Med. 1952;48:336-8.

22. Yeoh CB, Ford JM, Garret R. Tuberculous pseudoaneurysm of descending thoracic aorta. Surgical treatment. Arch Surg. 1963;86:318-22.

23. Silbergleit A, Arbulu A, Defever BA, Nedwicki EG. Tuberculous aortitis: surgical resection of ruptured abdominal false aneurysm. JAMA. 1965;193:333-5.

24. Garfein OB, Buckley MJ, Kurland GS. Postaortotomy myoctic pseudoaneurysm. A nonvalvular, potentially resectable source of intravascular sepsis following prosthetic valve surgery. JAMA. 1973;225:410-2.

25. Meyers BR, Shah R, Lefkowitz M. Mycotic aneurysm of the ascending aorta secondary to Serratia infection: differentiation from prosthetic valve endocarditis. Chest. 1974;65:215-7.

26. Atik FA, Navia JL, Svensson LG, Vega PR, Feng J, Brizzio ME. Surgical treatment of pseudoaneurysm of the thoracic aorta. J Thorac Cardiovasc Surg. 2006; $132: 379-85$.

27. Gin A, Hong H, Rosenblatt A, Black M, Ristow B, Popper R. Pseudoaneurysms of the mitral-aortic intervalvular fibrosa: survival without reoperation. Am Heart J. 2011;161:130.e1-5.

28. Lauer MS, Blackstone EH, Young JB, Topol EJ. Cause of death in clinical research: time for a reassessment? J Am Coll Cardiol. 1999;34:618-20.

Key Words: cardiovascular pseudoaneurysm, outcomes, surgery 